\title{
Comparativo del uso de Tecnologías de la Información y Comunicación (TIC) por Profesores de Dos Universidades Públicas de México
}

\author{
Agustín Lagunes-Domínguez, Carlos A.Torres-Gastelú, María A. Flores-García y \\ Armando Rodríguez-Figueroa \\ Universidad Veracruzana, Facultad de Contaduría y Administración, Campus Ixtac, Camino a Dos Ríos Km. \\ 1, Ixtaczoquitlán, México. (e-mail: aglagunes@uv.mx, ctorres@uv.mx,maflores@uv.mx; \\ zs08003001@estudiantes.uv.mx)
}

Recibido Jul. 20, 2014; Aceptado Sep. 25, 2014; Versión final recibida Nov. 22, 2014

\begin{abstract}
Resumen
El propósito de la investigación fue identificar las diferencias de uso de Tecnologías de la Información y Comunicación (TIC) por profesores adscritos a dos universidades públicas mexicanas. Para ello, se diseñó y aplicó un instrumento cuantitativo que buscaba identificar la frecuencia de uso de diez herramientas tecnológicas definidas. El análisis de los datos se realizó mediante valores de la estadística descriptiva calculadas por medio del software estadístico SPSS. Los resultados indican que no hay diferencias significativas entre las universidades en estudio. Se propone que se deben elaborar programas de educación continua para el profesorado como un elemento clave de actualización permanente de las tecnologías disponibles.
\end{abstract}

Palabras clave: tecnologías de la información y comunicación, TIC, enseñanza, era digital

\section{Comparison of the use of Information and Communication Technologies (ICT) by teachers of Two Public Universities in Mexico}

\begin{abstract}
The purpose of this research was the identification of differences in the use of Information and Communication Technologies (ICT) by teachers from two public universities in Mexico. For this, a quantitative instrument that sought to identify the frequency of use of ten defined technology tools was designed and applied. The analysis of the data was done using descriptive statistic included in the statistical software SPSS. The results indicate that there are no significant differences between the universities under study. It is proposed that universities must promote continuing education in their academic personnel as a key element for the permanent actualization of new compute technologies.
\end{abstract}




\section{INTRODUCCIÓN}

La incorporación de las Tecnologías de Información y Comunicación (TIC) en el mundo ha suscitado un proceso de unificación electrónica sin precedentes que ha afectado a las actividades y saberes de la humanidad. Por medio de ellas se han creado espacios virtuales que propician que las personas intercambien, interactúen y generen conocimientos, es a partir de esta tendencia que surge la relevancia de analizar las formas como se están llevando a cabo el uso de estas tecnologías en todos los ámbitos, pero particularmente en el medio universitario. Partiendo del hecho sobre la importancia de las TIC como un factor innegable en la vida del ser humano, se propone la premisa de analizar el uso de estas tecnologías en instituciones de educación superior en México, sobre todo, porque los profesores son agentes de cambio del aula, son ellos quienes en su función de líderes pueden lograr que la educación se transforme y por consecuencia, su país.

Para la UNESCO (2013) el uso de las TIC se aplica en la educación universitaria para elaborar materiales didácticos, exponer y compartir sus contenidos; propiciar la comunicación entre los alumnos, los profesores y el mundo exterior; elaborar y presentar conferencias; realizar investigaciones académicas; así como para brindar apoyo administrativo y matricular a los estudiantes. En el año de 2012, la UNESCO generó un documento estadístico llamado "Uso de TIC en educación en América Latina y el Caribe" con el propósito de mostrar cómo se encontraba esta región en cuanto a la penetración de las TIC y las políticas nacionales. En dicho documento se muestra que 31 de 38 países (82\%) tienen al menos un tipo de definición formal de su TIC en iniciativas de educación; mientras que 9 de ellos (24\%) tienen todas las definiciones formales. Entre estos últimos se incluyen Anguila, Bahamas, Barbados, Chile, Ecuador, Guatemala, San Vicente y las Granadinas, Uruguay y Venezuela. Por el contrario, Curazao, Dominica, Montserrat y Surinam no tienen definiciones oficiales o instituciones reglamentarias en materia de TIC en la educación (UNESCO, 2012).

Por lo que se refiere a los profesores y las TIC en educación, en el mismo documento la UNESCO indica que ellos son considerados con frecuencia como la influencia externa más importante en el aprendizaje en el aula y en la educación en general. Como tales, los profesores desempeñan un papel invaluable para asegurar que las TIC se utilicen de manera efectiva por los alumnos, dentro y fuera del aula. Los profesores no sólo necesitan saber cómo instruir a los alumnos a utilizar las TIC de manera eficaz, sino que también necesitan ser entrenados para usarlas con el fin de enseñar los diversos temas de manera efectiva. El cómo se logra esto varía de un país a otro, sin embargo, dadas las políticas muy dispares y la diversidad de planes de estudio sobre la mejor manera de integrar las TIC y aplicarlas en el aula, hace que esto se torne complejo (UNESCO, 2012).

Por lo que se refiere a México, la Asociación Nacional de Universidades e Instituciones de Educación Superior (ANUIES) desde su fundación en 1950, ha participado en la formulación de programas, planes y políticas nacionales, así como en la creación de organismos orientados al desarrollo de la educación superior mexicana. La Asociación está conformada por 165 universidades e instituciones de educación superior, tanto públicas como particulares de todo el país, que atienden al $80 \%$ de la matrícula de alumnos que cursan estudios de licenciatura y de postgrado (ANUIES, 2012). ANUIES generó un libro llamado "Documento estratégico para la innovación en la Educación Superior" y dentro de sus apartados señala dos elementos en los que se apoya esta investigación: el docente y su papel en la innovación y las tecnologías para la innovación educativa que muestran el camino a seguir en estos temas en la Educación Superior en México.

Para el uso de las TIC es necesario diseñar y ofrecer programas de formación con el fin de facilitar la incorporación de las TIC en la práctica docente (Valcárcel y Cantón Mayo, 2009). Es recomendable que los profesores procuren diseñar actividades en las que a través del uso de las tecnologías les indiquen a los alumnos los objetivos y procedimientos para que luego el estudiante pueda hacerlo de manera autónoma como una estrategia para mejorar el rendimiento académico (García-Valcárcel y Arras, 2011). Una vez que las universidades deciden implementar las TIC en el proceso de enseñanza - aprendizaje, los profesores deben capacitarse con personas expertas en el funcionamiento de las tecnologías que se van a implementar en su universidad. Por su parte, los profesores también tendrán que procurar por si mismos la forma de estar en constante capacitación, no sólo con todo aquello que se relacione con la tecnología en general, sino también, de manera específica, con respecto a los medios que realmente necesite, ya que en la actualidad la tecnología abarca cada vez más disciplinas y los profesores no pueden quedarse con los conocimientos ya adquiridos anteriormente. En este ámbito de ideas, Valcárcel y Cantón Mayo (2009) mencionan que las competencias más importantes que deben adquirir los profesores son: saber utilizar Internet para buscar información y recursos para la preparación de sus clases; así como para conocer sitios tales como portales, páginas web, revistas electrónicas, diccionarios y buscadores, sobre su especialidad, entre otros. Saber utilizar las principales herramientas electrónicas de Internet para comunicarse, como por ejemplo el correo electrónico, las listas de distribución y los foros. Y de igual manera, saber utilizar programas informáticos específicos de su campo profesional. 
En cuanto a la competencia de los profesores se han realizado estudios como el de Cardoso et al. (2013) sobre docentes en posgrados de administración y otros como el de Rocha (2013) quieren medir la efectividad de la docencia de los profesores en Educación Superior desde la perspectiva de los estudiantes. Desde esa misma perspectiva Muñoz-Cano et al. (2011) muestran las dificultades y facilidades del uso de las TIC. Así mismo Marulanda et al. (2014) se enfocan al acceso y uso de las TIC en los estudiantes preuniversitarios en Colombia y estudios más específicos como el de Hernández et al. (2014) se enfocan al uso de las TIC en alguna experiencia educativa como en este caso la química orgánica.

Se han realizado diversas investigaciones sobre la combinación TIC y profesores, algunas enfocadas a las competencias que deben poseer como las "ICT Skills for Guidance Counsellors" integradas en el "Programa Leonardo Da Vinci" de la Unión Europea, otras como la de Suárez-Rodríguez et al. (2012) dónde analizan la influencia de factores personales y contextuales clave para el uso de las TIC y, desde luego, en México con las reformas en los niveles educativos, se ha escrito para aprovechar dichos cambios y determinar el uso de las TIC como lo investigan Burrola y Vera (2013). Así mismo, se ha investigado sobre la formación de los profesores enfocándose a los aspectos fundamentales de la formación del profesorado en TIC (Llorente Cejudo, 2008; Sarmiento y Guillén 2008; López y Chávez, 2013). Por otro lado, Fainholc Nervi et al. (2013) apuntaron a su uso pedagógico.

Otros autores se han enfocado al diseño y aplicación de programas de capacitación en TIC para áreas específicas, tal como lo hicieron en México Farías et al. (2013) en "Gestión de un programa de capacitación en línea para el desarrollo de habilidades y capacidades TIC en profesores de negocios". Existen estudios comparativos como el de Rosario Noguera y Vásquez Melo (2012) quienes indagaron sobre la formación que dice tener un docente universitario en el uso de las Tecnologías de la Información y Comunicación en ambientes educativos y para ello aplicaron un cuestionario a los docentes de dos instituciones de educación superior: la Universidad de Carabobo (Universidad Pública) y la Universidad Metropolitana (Universidad Privada) en Venezuela, el resultado entre la privada versus la pública, demostró que en la primera sus profesores tienen una buena preparación, mientras que en la privada fue regular.

En cuanto a universidades privadas, Chumpitaz Campos y Rivero Panaqué (2012) realizaron un estudio sobre una de ellas en Lima acerca del uso pedagógico de las TIC y entre los resultados que obtuvieron fueron que el $94,7 \%$ de los encuestados confirma utilizar herramientas tecnológicas en sus clases, de los cuales el $45 \%$ las usan todos los días. Por lo que se refiere a las universidades públicas que son el objeto de esta investigación, Fischman (2008) en su ensayo, hace un recorrido por las expectativas y a lo que llama crisis de estas universidades en el siglo XXI; en este punto, Bulchand-Gidumal y Melian-Gonzalez (2009) coinciden en que las universidades públicas deben rediseñarse y considerar el soporte que dan a sus profesores y estudiantes en cuanto a las TIC. Por otra parte, Losada Iglesias et al. (2012) analizaron la situación de la tecnología educativa en los grados de educación en universidades españolas tras la implantación del Espacio Europeo de Enseñanza Superior, tomaron 48 del total de 76 universidades públicas existentes encontrando que $87 \%$ de los analizados ofrecen la posibilidad de formar, en mayor o menor medida en competencias TIC.

En relación a estudios sobre análisis comparativos en diferentes regiones de México, Arras, Torres y Fierro (2012) revisaron las perspectivas de los alumnos hacia sus competencias en TIC en dos universidades públicas una localizada en el norte y otra en el sur. Sus resultados se concentran en las actitudes y en el grado de dominio que dicen poseer, soslayando la medición de los conocimientos reales que poseen. El trabajo exploratorio de estos autores resalta que los alumnos se atribuyen una competencia elevada presentando similitudes en ambas universidades, sin embargo no se abordó la postura de los profesores.

Ahora bien, la relevancia de la realización de análisis comparativos está dada por la inequidad en el desarrollo económico y social de México. Por tanto, la distribución de la riqueza y población se manifiesta en polos de desarrollo económicos con diferentes capacidades productivas y agrícolas. En estos términos se puede clasificar al país en dos grandes bloques: Norte y Sur. El Norte caracterizado por un mayor desarrollo económico impulsado por la cercanía con Estados Unidos, mejores niveles de vida, menor índice de población y mayor efectividad en la explotación de sus recursos.

En tanto el Sur presenta un atraso social significativo, mayores niveles de pobreza, de mortalidad infantil, con una mayor concentración de la población indígena por tanto población rural es mucho más importante (Dávila, Kessel y Levy, 2002). El atraso relativo de la región se refleja también en los indicadores de desarrollo productivo, por tanto las políticas públicas pueden revertir esta situación a través de la realización de estudios de índole comparativo que proporcionen información útil para este propósito. La literatura ha señalado la relevancia de la realización de estudios comparativos como un mecanismo para detectar necesidades y generar políticas tecnológicas internas en las universidades, así como en la emisión de políticas públicas nacionales que orienten el rumbo de la educación en México. En este sentido, el presente trabajo pretende abonar al conocimiento presentando los resultados de un estudio comparativo entre dos 
universidades públicas mexicanas: una localizada en el norte y otra en el sur. Una aportación clave está dada en términos de identificar la percepción en este caso de los profesores hacia el uso de las TIC.

\section{MATERIALES Y MÉTODOS}

La estrategia metodológica consistió en realizar una investigación cuantitativa de corte descriptivo aplicando una encuesta. Para ello se estableció una muestra por conveniencia considerando a dos universidades públicas, una del sur y otra del norte de México. El instrumento para esta investigación fue valorado por 10 expertos en coincidencia con Chacón Víquez (2013), se recibieron los cambios sugeridos, se realizaron las correcciones y una vez que los expertos estuvieron de acuerdo se liberó el instrumento para su aplicación. La aplicación de la prueba de Alfa Cronbach fue de $80 \%$, indicando con ello un valor aceptable de confiabilidad.

Se aplicó a los profesores de ambas universidades un instrumento de 22 preguntas divididas en 14 secciones, el cual fue validado por investigadores expertos, tanto en TIC como y en la elaboración de instrumentos. Se utilizó la fórmula del muestreo aleatorio simple para poblaciones finitas de Münch Galindo y Angeles (1998) dicha fórmula permite determinar el tamaño de la muestra y queda representada de la siguiente manera:

$$
\eta_{\theta}=\frac{\sigma^{2} Z_{(\infty / 2)}^{2}}{e^{2}}
$$

La universidad del Norte cuenta con 436 profesores y se tomó una muestra de 30 . Por lo que se refiere a la universidad del Sur, son 60 profesores, encuestándose a 18 de ellos. La selección de la muestra en ambas universidades fue probabilística. La distribución de la muestra quedó de la siguiente manera: 40\% fueron mujeres y $60 \%$ varones. En relación a la formación universitaria que habían recibido los profesores: $23 \%$ Administrador de Empresas o carrera a fin; 27\% Contador Público; 15\% Sistemas o carrera afín con computación; $12 \%$ Abogados; $8 \%$ ingenieros y el resto de otra profesión. Por lo que se refiere a su antigüedad laboral el promedio de los profesores acumulaba 17 años. En tanto, el de más antigüedad contaba con 37 años, y el de menor 2 años.

En cuanto a la validación de la captura esta se logro mediante tres capturas por separado y posteriormente la unión de las mismas en una hoja de cálculo aplicando la formula:

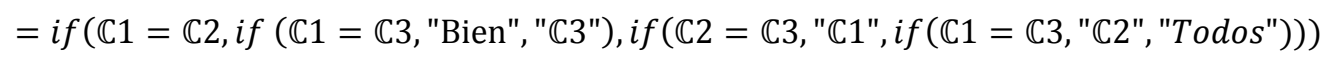

La fórmula indica cuál de las capturas es diferente, "Todos" si las tres están diferentes; "C1", "C2" o "C3" si alguna de ellas está diferente; el tercer caso es "Bien" cuando todas coinciden. En el primer y segundo caso, se recurre al instrumento original para corregir y que todas los reactivos de la captura indiquen "Bien".

Una vez determinada la validez de la captura, se importaron los datos en SPSS, donde para comprobar la hipótesis nula, se calculó Chi cuadrada tomando en cuenta las variable universidad y uso de las TIC.

\section{RESULTADOS Y DISCUSIÓN}

Para analizar los resultados se presentan dos tablas, la frecuencia con la que utilizan los profesores las TIC en sus clases y el porcentaje de su uso. Posteriormente, se presenta un concentrado comparando los resultados obtenidos entre ambas universidades.

Tabla 1: Frecuencia del uso de las TIC en clases de ambas universidades.

\begin{tabular}{|l|r|r|r|r|}
\hline \multirow{2}{*}{} & \multicolumn{2}{|c|}{ Universidad del Norte } & \multicolumn{2}{c|}{ Universidad del Sur } \\
\cline { 2 - 5 } & Porcentaje & $\begin{array}{r}\text { Porcentaje } \\
\text { acumulado }\end{array}$ & Porcentaje & $\begin{array}{r}\text { Porcentaje } \\
\text { acumulado }\end{array}$ \\
\hline Frecuentemente & 76.7 & 76.7 & 61.1 & 61.1 \\
\hline A veces & 20.0 & 96.7 & 27.8 & 88.9 \\
\hline Nunca & 3.3 & 100.0 & 0 & 88.9 \\
\hline No aplica & 0 & 100.0 & 11.1 & 100.0 \\
\hline Total & 100.0 & & 100.0 & \\
\hline
\end{tabular}


La Tabla 1 indica que el $96.7 \%$ de los profesores que se encuestaron en la universidad del Norte recurren a los medios tecnológicos como un apoyo alternativo para la impartición de sus clases, quedando solo en $3.3 \%$ que no las utiliza. En correspondencia se muestra que en la universidad del Sur el $88.9 \%$ utiliza las TIC con cierta frecuencia, aunque nadie respondió que no las utiliza.

Tabla 2: Porcentaje que utilizan las TIC en sus clases de ambas universidades.

\begin{tabular}{|l|r|r|r|r|}
\hline & \multicolumn{2}{|c|}{ Universidad del Norte } & \multicolumn{2}{c|}{ Universidad del Sur } \\
\hline & Porcentaje & $\begin{array}{c}\text { Porcentaje } \\
\text { acumulado }\end{array}$ & Porcentaje & $\begin{array}{r}\text { Porcentaje } \\
\text { acumulado }\end{array}$ \\
\hline $0 \%$ & 6.7 & 6.7 & 0 & 0 \\
\hline $10 \%$ & 0 & 6.7 & 5.6 & 5.6 \\
\hline $20 \%$ & 3.3 & 10.0 & 5.6 & 11.1 \\
\hline $30 \%$ & 3.3 & 13.3 & 16.7 & 27.8 \\
\hline $40 \%$ & 3.3 & 16.7 & 0 & 27.8 \\
\hline $50 \%$ & 0 & 16.7 & 16.7 & 44.4 \\
\hline $60 \%$ & 6.7 & 23.3 & 11.1 & 55.6 \\
\hline $70 \%$ & 6.7 & 30.0 & 22.2 & 77.8 \\
\hline $80 \%$ & 33.3 & 63.3 & 5.6 & 83.3 \\
\hline $90 \%$ & 20.0 & 83.3 & 11.1 & 94.4 \\
\hline $100 \%$ & 16.7 & 100.0 & 5.6 & 100.0 \\
\hline Total & 100.0 & & 100.0 & \\
\hline
\end{tabular}

A la pregunta en qué porcentaje utilizan las TIC en la universidad del Norte, el grosor de los profesores respondieron que entre el $80 \%$ y el $90 \%$ las utilizan en sus clases, siendo un acumulado del $53.3 \%$ de los profesores encuestados. El $22.2 \%$ de los profesores de la universidad del Sur respondieron que utilizan las TIC en un $70 \%$ de sus clases, mientras que el $16.7 \%$ manifestó que sólo en el $50 \%$ de las mismas. En estas dos comparaciones se visualiza la diferencia entre la universidad del Norte con respecto a la del Sur. Del instrumento aplicado a profesores se listaron una serie de herramientas y ellos seleccionaron con qué frecuencia las utilizaban, en una escala de: nunca, rara vez, una vez a la semana, dos veces a la semana, tres veces a la semana, siempre o no aplica cuando no cuentan con dicha herramienta en su universidad.

Para la Tabla 3 se tomaron las dos frecuencias más altas y se realizó el acumulativo; posteriormente, se calculó la diferencia de uso de cada herramienta, entre las universidades. En particular, la Tabla 3 indica que en la universidad del Norte utiliza en mayor medida el video, el proyector, Internet y los simuladores, mientras que la del Sur la plataforma educativa, videos, chat, correo electrónico, blogs y página personal. Mención especial merece el correo electrónico, ya que la diferencia es significativa entre el Sur y el Norte.

Tabla 3: Comparativo del uso de las herramientas tecnológicas entre ambas universidades.

\begin{tabular}{|l|l|r|r|c|c|}
\hline \multirow{2}{*}{} & \multirow{2}{*}{ Frecuencia } & \multicolumn{2}{l}{ Utilización en porcentaje } & \multicolumn{2}{|c|}{ Diferencia en porcentaje } \\
\cline { 3 - 6 } & & Norte & Sur & Norte & Sur \\
\hline Video Proyector & Frecuentemente & 73.3 & 66.7 & 6.6 & 0 \\
\hline Internet & Frecuentemente & 60.0 & 44.4 & 15.6 & 0 \\
\hline Plataforma educativa & Frecuentemente & 13.3 & 16.7 & 0 & 3.4 \\
\hline Simuladores & Frecuentemente & 16.7 & 0.0 & 16.7 & 0 \\
\hline Videos & Frecuentemente & 20.0 & 22.2 & 0 & 2.2 \\
\hline Audios & Frecuentemente & 16.7 & 16.7 & 0 & 0 \\
\hline Chat & Frecuentemente & 23.4 & 33.4 & 0 & 10 \\
\hline Correo electrónico & Frecuentemente & 56.7 & 88.9 & 0 & 32.2 \\
\hline Blog & Frecuentemente & 6.7 & 16.7 & 0 & 10 \\
\hline Sitio web personal & Frecuentemente & 13.3 & 16.7 & 0 & 3.4 \\
\hline \multicolumn{7}{l}{} & & Total & 38.9 & 61.2 \\
\hline
\end{tabular}


Analizando la tabla 3 se considera "frecuentemente" cuando utilizan la herramienta más de tres veces a la semana, adicionalmente se cuestionó a los profesores sobre la capacitación y el 100\% contestó que necesitaba capacitación, se advierte la concordancia con Valcárcel y Cantón Mayo (2009) en la necesidad de diseñar cursos de formación para facilitar la incorporación de las TIC en la práctica docente.

La parte medular de la investigación se refiere a comprobar la hipótesis nula: "Existen similitudes en la percepción de los profesores hacia su uso de las TIC en la Universidad del Norte con respecto a la universidad seleccionada del Sur". En tanto, la hipótesis alterna fue: "No existen similitudes en la percepción de los profesores hacia su Uso de las TIC en la Universidad del Norte con respecto a la universidad seleccionada del Sur". Para ello se tomaron en cuenta las medias y se calculó Chi Cuadrada con un nivel de $95 \%$ de confianza, se rechazaba Ho si el valor de $\mathrm{P}$ que resultara de la prueba fuese menor a 0.05 , mientras que el valor obtenido fue 0.085 , considerablemente mayor, por lo cual se concluye que se comprueba la hipótesis nula "Existen similitudes en la percepción de los profesores hacia su uso de las TIC en la Universidad del Norte con respecto a la universidad seleccionada del Sur" y que la creencia que las universidades del norte utilizan más las TIC no se cumple al menos para el caso de las universidades consideradas para este estudio.

\section{CONCLUSIONES}

De acuerdo a la consideración inicial, se puede afirmar que de acuerdo a lo contestado por los profesores de las universidades de ambas regiones, los profesores de la universidad del Norte utilizan de manera similar las TIC en relación a la del Sur, como lo demuestra la prueba Chi Cuadrada y que esta última utiliza de forma más diversificada las TIC como lo muestra la Tabla 3.

Aunque sí se puede concluir que hay una diferencia significativa entre las herramientas que se utilizan en la universidad del Norte con referencia a la del Sur, en la primera el porcentaje de uso de los profesores respondieron que entre el $80 \%$ y el $90 \%$ las utilizan en sus clases, de hecho la diferencia a favor de la del Sur es el correo electrónico con un mayor uso en $32 \%$, mientras que la del Norte utiliza más los simuladores siendo que la del Sur no los usa, esto refleja que la universidad del Sur utiliza una herramienta común, mientras que la del Norte cuenta con los recursos para adquirir simuladores.

Existe una brecha entre ambas universidades, coincidiendo en que no están explotando adecuadamente las TIC, contribuyendo así a la continuación de las clases tradicionales, si se complementaran en su uso ambas regiones se lograría un mejor aprovechamiento de ellas en las universidades públicas.

Cabe señalar que ambas universidades han implementado proyectos institucionales que procuran cubrir las deficiencias en términos de cobertura en la infraestructura tecnológica como en cursos de computación sobre algunas de las herramientas tecnológicas. Sin embargo, una debilidad que se ha detectado tiene que ver con la ausencia de cursos-taller que estén orientados a aplicar los conocimientos técnicos y pedagógicos en la incorporación de actividades didácticas que propicien el tránsito a una incorporación de las TIC en el modo presencial, pero incluso en otras modalidades como la semi-presencial o virtual.

Debido a la poca utilización de las TIC se recomienda a las universidades la elaboración de un plan estratégico que incentive la incorporación gradual y efectiva desde sus programas de estudio hasta el diseño instruccional de cada profesor.

La recomendación se orienta en elaborar programas de certificación y actualización de conocimientos tecnológicos y pedagógicos en el profesorado universitario como un elemento clave en el proceso de formación continua que debe ser promovido por las universidades públicas mexicanas como un mecanismo para transitar hacia una educación que apañe a la brecha digital.

\section{REFERENCIAS}

ANUIES. Recuperado el 4/12/2012, de ANUIES: http://www.anuies.mx/la_anuies/que_es/laanuies.php (2012).

ANUIES. Documento estratégico para la innovación en la educación superior (2a Edición ed.). DF, México (2004).

Arras, A.M., Torres-Gastelú, C.A., y Fierro, L.E. Competencias en TIC y rendimiento académico en las universidades autónoma de Chihuahua y Veracruzana. México. Pearson (2012).

Bulchand-Gidumal, J., y Melian-Gonzalez, S. Redesign of the IS/ICT help desk at a Spanish public university. High Educ (60), 205-216 (2010). 
Burrola, M., y Vera, J. Á. Study about ICT skills in junior high school teachers under Mexico's educational reform. International Journal of Psychological Research, 6 (2), 59-70 (2013).

Cardoso E. O., Cerecedo M. T. y Venegas E. A. Las Competencias Docentes en los Programas de Posgrado en Administración. Un Estudio de Diagnóstico. doi: 10.4067/S0718-50062013000200006, Formación Universitaria. Vol. 6(2), 43-50 (2013).

Chacón Víquez L.D. Diseño, construcción y validación de un instrumento para la evaluación de las competencias de los docentes (IECOD) desde la perspectiva del alumno, en el Instituto Superior Tecnológico Jesús de Nazareth, San Pedro de Sula, Honduras. Revista Calidad en la Educación Superior, 4(1), 96-129 (2013).

Chumpitaxz, L., y Rivero, C. Uso cotidiano y pedagógico de las TIC por profesores de una universidad privada de Lima. Educación, XXI (41), 81-100 (2012).

Dávila, E., Kessel, G. y Levy, S. El sur también existe: un ensayo sobre el desarrollo regional de México. Economía Mexicana NUEVA ÉPOCA, volumen XI, número 2, México. (2002).

EEES. Recuperado el 4/12/2012, de http://www.eees.es/es/eees-estructuras-educativas-europeas (2008).

Fainholc, B., Nervi, H., Romero, R., y Halal, C. La formación del profesorado y el uso pedagógico de las TIC. RED. Revista de Educación a Distancia (38), 1-14 (2013).

Farías, G., Pedraza, N., y Lavín, J. Gestión de un programa de capacitación en línea para el desarrollo de habilidades y capacidades TIC en profesores de negocios. Revista Electrónica de Investigación Educativa, 15 (1), 45-61 (2013).

Fischman, G. E. Las Universidades Públicas en el Siglo XXI. Grandes expectativas, algunas promesas y muchas incertidumbres. Universitas humanística , 239-270 (2008).

García-Valcárcel, A. y Arras, A. Competencias en TIC y rendimiento académico en la universidad. Diferencias por género. México. Pearson (2011).

Hernández M. R., Rodríguez V. M., Parra F. J. y Velázquez P. Las Tecnologías de la Información y la Comunicación (TICs) en la Enseñanza-Aprendizaje de la Química Orgánica a través de Imágenes, Juegos y Videos. doi: 10.4067/S0718-50062014000100005, Formación Universitaria. Vol. 7(1), 31-40 (2014).

Llorente, M. Aspectos fundamentales de la formación del profesorado en TIC. Pixel-Bit. Revista de Medios y Educación (31), 121-130 (2008).

López, M. C., y Chávez, J. A. La formación de profesores universitarios en la aplicación de las TIC. Revista Electrónica Sinéctica (41), 1-18 (2013).

Losada, D., Valverde, J., y Correa, J. M. La tecnología educativa en la universidad pública española. PixelBit. Revista de Medios y Educación (41), 133-148 (2012).

Marulanda C. E., Giraldo J. y López M. Acceso y uso de las Tecnologías de la información y las Comunicaciones (TICs) en el aprendizaje. El Caso de los Jóvenes Preuniversitarios en Caldas, Colombia. doi: 10.4067/S0718-50062014000400006, Formación Universitaria. Vol. 7(4), 47-56 (2014).

Muñoz-Cano J. M., Córdova J. A. y Priego H. Dificultades y facilidades para el desarrollo de un proceso de innovación educativa con base en las Tecnologías de la Información y Comunicación (TIC). doi: 10.4067/S0718-50062012000100002, Formación Universitaria. Vol. 5(1), 3-12 (2012).

Münch, L., y Angeles, E. Métodos y técnicas de investigación. México: Trillas (1998).

OEA. Recuperado el 04 de 12 de 2012, de OEA: http://www.oas.org/es/acerca/quienes_somos.asp (2012).

Rocha R. Escala de Opinión de los Estudiantes sobre la Efectividad de la Docencia (EOEED) en Educación Superior. doi: 10.4067/S0718-50062013000600003, Formación Universitaria. Vol. 6(6), 13-22 (2013).

Rosario, H. J., y Vásquez, L. F. Formación del docente universitario en el uso de TIC. Caso universidades públicas y privadas. (U. de Carabobo y U. Metropolitana). Pixel-Bit. Revista de Medios y Educación (41), 163-171 (2012).

Sarmiento, M., y Guillén, J. Formacion en TIC: Necesidad del profesor universitario. Laurus revista de educación, 14 (28), 11-34 (2008). 
Suárez-Rodríguez, J. M., Almerich, G., Díaz-García, I., y Fernández-Piqueras, R. Competencias del profesorado en las TIC. Influencia de factores personales y contextuales. Universitas Psychologica, 11 (1), 293-309 (2012).

UNESCO. Recuperado el 04 de 12 de 2012, de

http://unesdoc.unesco.org/images/0014/001473/147330s.pdf (2009).

UNESCO. Higher Education. Recuperado el 5 de 07 de 2014, de Sitio UNUESCO:

http://www.unesco.org/new/es/unesco/themes/icts/lifelong-learning/higher-education/ (2013).

UNESCO. ICT in education in Latin America and the Caribbean a regional analysis of ICT integration and ereadiness. Montreal, Quebec, Canada (2012).

Valcárcel, A. G., y Cantón, I. La Incorporación de las TIC en la Docencia Universitaria: recursos para la formación del profesorado. Barcelona: Davinci Continental S.L. (2009). 